\title{
The predictive value of early molecular response in chronic myeloid leukaemia patients treated with imatinib in a single real-world medical centre in a developing country
}

Ping Chong Bee ${ }^{1}$, MMed, Veera Sekaran ${ }^{2}$, MPath, Richard Rui Jie $\mathrm{Ng}^{1}$, MBBS, Ting Yi Kweh ${ }^{1}$, MBBS,

Gin Gin Gan $^{1}$, MBBS, FRCP

INTRODUCTION The prognosis of patients with chronic myeloid leukaemia (CML) has improved since the introduction of imatinib. However, patients who do not achieve complete cytogenetic response (CCyR) and major molecular response (MMR) have poorer prognosis. Recent clinical trials have demonstrated that early and deeper cytogenetic and molecular responses predict a better long-term outcome. This study aimed to analyse the relationship between early molecular response and clinical outcome in a real-life setting.

METHODS This retrospective study included all patients with CML, in chronic or accelerated phase, who were treated with imatinib at University of Malaya Medical Centre, Malaysia.

RESULTS A total of 70 patients were analysed. The median follow-up duration was 74 months, and the cumulative percentages of patients with CCyR and MMR were $80.0 \%$ and $65.7 \%$, respectively. Overall survival (OS) and event-free survival (EFS) at ten years were $94.3 \%$ and $92.9 \%$, respectively. Patients who achieved CCyR and MMR had significantly better OS and EFS than those who did not. At six months, patients who had a BCR-ABL level $\leq 10 \%$ had significantly better OS and EFS than those who had a BCR-ABL level > 10\%. The target milestone of CCyR at 12 months and MMR at 18 months showed no survival advantage in our patients.

CONCLUSION Our data showed that imatinib is still useful as first-line therapy. However, vigilant monitoring of patients who have a BCR-ABL level $>10 \%$ at six months of treatment should be implemented so that prompt action can be taken to provide the best outcome for these patients.

Keywords: chronic myeloid leukaemia, complete cytogenetic response, early major molecular response, imatinib, survival

\section{INTRODUCTION}

Chronic myeloid leukaemia (CML) is now considered an indolent disease. This was achieved after imatinib mesylate, a selective BCR-ABL tyrosine kinase inhibitor (TKI), was introduced for the treatment of CML a decade ago. In fact, $90 \%$ of $\mathrm{CML}$ patients on imatinib mesylate treatment are still alive after five years. ${ }^{(1)}$ Furthermore, patients who achieve complete cytogenetic response (CCYR) and major molecular response (MMR) by 12 and 18 months, respectively, show significantly better progression-free survival (PFS). ${ }^{(2)}$ Nevertheless, there is still a substantial number of patients who do not achieve the desired response. An eightyear follow-up conducted in the Immediate Risk Stratification Improves Survival (IRIS) trial showed that $37 \%$ of the patients on imatinib had an unfavourable outcome, with $32 \%$ of them failing to achieve CCyR or losing CCyR, and 5\% of them being intolerant to imatinib. ${ }^{(3)}$

Many studies have demonstrated that the achievement of early CCYR and MMR among imatinib-treated CML patients is associated with improved survival. ${ }^{(4-7)}$ Based on the findings of these studies, molecular response at defined time points is now widely accepted as a predictive marker for subsequent outcomes in CML patients. Second-generation TKIs, such as dasatinib, nilotinib and bosutinib, are more potent than imatinib, and have proven efficacy in patients who are resistant or intolerant to imatinib. ${ }^{(8,9)}$ More patients have achieved optimal cytogenetic and molecular responses when treated with these newer drugs than with imatinib. Furthermore, the responses achieved using the newer drugs were faster and deeper, contributing to a lower rate of disease progression and superior long-term outcomes. ${ }^{(10-12)}$

Both the United States' National Comprehensive Cancer Network (NCCN) and the European LeukemiaNet (ELN) updated their guidelines to include $\mathrm{BCR}-\mathrm{ABL} \leq 10 \%$ as a treatment response milestone at three months. ${ }^{(13,14)}$ ELN defines optimal response as a BCR-ABL level $<1 \%$ at six months and $\leq 0.1 \%$ at 12 months, while NCCN defines it as CCyR, with or without MMR, by $12-18$ months. In addition, a BCR-ABL level $>10 \%$ at six months and $>1 \%$ at 12 months is defined by ELN as treatment failure that requires a switch of TKIs. On the other hand, NCCN recommends a change to alternate TKIs if the BCR-ABL levels are $>10 \%$ at three months or if Philadelphia (Ph) chromosomepositive cells are present at 12 months.

As Malaysia is a developing country without comprehensive medical insurance coverage, most CML patients in Malaysia are dependent on free imatinib provided through the Malaysia Patient

${ }^{1}$ Department of Medicine, ${ }^{2}$ Department of Pathology, University of Malaya Medical Centre, Kuala Lumpur, Malaysia

Correspondence: Dr Bee Ping Chong, Consultant Haematologist, Department of Medicine, Faculty of Medicine, University of Malaya, 50603 Kuala Lumpur, Malaysia. bpingchong@gmail.com 
Assistance Programme. It is extremely difficult for the treating haematologist to adjust the treatment protocol according to the international guidelines, as the majority of patients are unable to afford the cost of newer TKIs. Therefore, the objectives of the present study were to: (a) analyse the outcome of treatment with imatinib; and (b) confirm if early achievement of molecular responses according to the ELN and NCCN guidelines predicted better survival in CML patients who were treated with imatinib. The findings of this study would help to shed light on the outcomes of $\mathrm{CML}$ patients who are not treated according to the ideal protocol (i.e. the guidelines recommended by ELN and NCCN).

\section{METHODS}

This observational, retrospective study included all patients who were diagnosed with chronic- or accelerated-phase CML and treated with imatinib at University of Malaya Medical Centre, Kuala Lumpur, Malaysia. The patients' characteristics, molecular and cytogenetic responses to imatinib, and survival probabilities were examined.

The patients' cytogenetic response was based on the prevalence of $\mathrm{Ph}$ chromosome-positive metaphases among at least 20 metaphases investigated in each bone marrow sample. Cytogenetic response was defined as: (a) complete, if there were $0 \% \mathrm{Ph}$ chromosome-positive cells in metaphase; (b) partial, if $1 \%-35 \%$ were $\mathrm{Ph}$ chromosome-positive cells in metaphase; and (c) non-existent, if $>35 \%$ were $\mathrm{Ph}$ chromosome-positive cells in metaphase. Both complete and partial cytogenetic responses (i.e. $\mathrm{Ph}$ chromosome-positive cells $=0 \%-35 \%$ ) were considered as major cytogenetic responses in this study. Molecular monitoring was done by measuring the number of BCR-ABL transcripts in peripheral blood. This was done using reverse transcription-polymerase chain reaction (RT-PCR) in our laboratory, which is compliant with international standards. MMR was defined as a BCR-ABL/ABL ratio $\leq 0.1 \%$, while complete molecular response referred to the absence of detectable BCR-ABL transcripts via RT-PCR, at a sensitivity $>10^{-4.5}$.

The endpoints we examined were death from any cause during treatment, disease progression into more advanced phases, and loss of complete haematologic and/or major cytogenetic responses. The duration of response was calculated from the first reported date of response to the earliest date of reported relapse or death. Time to progression was defined as the time from the start of the treatment to the onset of: (a) an accelerated or a blastic phase; (b) discontinuation of therapy due to unsatisfactory therapeutic effects; or (c) death. Survival was calculated from the beginning of therapy until the time of death from any cause.

SPSS for Windows version 16.0 (SPSS Inc, Chicago, IL, USA) was used to process, manage and analyse the data to determine the factors that significantly affect the response rate, survival and frequency of adverse effects. Frequencies and descriptive statistics were also generated using the same program. Differences among the variables were evaluated using chi-square test, and survival probabilities were estimated using Kaplan-Meier's method. A p-value $<0.05$ was considered statistically significant.
Table I. Demographics of the patients $(n=70)$.

\begin{tabular}{lc}
\hline Parameter & No. (\%) \\
\hline Gender & $49(70.0)$ \\
Male & $21(30.0)$ \\
Female & \\
Ethnicity & $25(35.7)$ \\
$\quad$ Malay & $39(55.7)$ \\
Chinese & $6(8.6)$ \\
Indian & $44(30-55)$ \\
Age at CML diagnosis* (yr) & $45.5(30.8-56.2)$ \\
Age at start of treatment* (yr) & \\
Phase of CML at diagnosis & $65(92.9)$ \\
Chronic phase & $5(7.1)$ \\
$\quad$ Accelerated phase & $66(94.3)$ \\
Patient survival & $74(15-197)$ \\
Duration of follow-up* (mth)
\end{tabular}

*Data presented as median (interquartile range). CML: chronic myeloid leukaemia

\section{RESULTS}

A total of 70 patients were included in the study $-65(92.9 \%)$ in chronic phase and $5(7.1 \%)$ in accelerated phase (Table I). The median follow-up duration was 74 (range 15-197) months. The cumulative number of patients with CCyR and MMR were $56(80.0 \%)$ and $46(65.7 \%)$, respectively. $28(50.0 \%)$ of the 56 patients who achieved CCyR did so at or before 12 months of treatment, while the remainder achieved CCyR after 12 months of treatment. 21 (45.7\%) of the 46 patients who achieved MMR did so at or before 18 months of treatment, while the remainder achieved MMR after 18 (range 19-120) months of treatment.

The overall survival (OS) at ten years was $94.3 \%$ (Fig. 1). The OS was significantly better among the patients who achieved CCyR than among those who did not (98.2\% vs. $75.0 \%$, respectively; $p<0.001$ ) (Fig. 2 \& Table II). Similarly, the OS of the patients who achieved and maintained MMR was significantly better than that of the patients who did not $(97.8 \%$ vs. $87.1 \%$, respectively; $p=0.012$ ) (Fig. $3 \&$ Table II). When the patients were stratified according to when they achieved CCYR and MMR, the OS of the patients who achieved CCyR at or before 12 months was $100.0 \%$, while the OS of those who achieved CCYR after 12 months was $95.8 \%$ ( $p=0.637)$, and the OS of the patients who achieved MMR at or before 18 months was $100.0 \%$, while the OS of those who achieved MMR after 18 months was $97.8 \%(p=0.190)$.

Patients who had a BCR-ABL level $\leq 10 \%$ at six months had significantly better OS than those who had a BCR-ABL level $>10 \%$ at six months $(100.0 \%$ vs. $88.9 \%$, respectively; $p=0.041)$ (Fig. 4 \& Table II). However, BCR-ABL level at three months did not predict better OS; patients who had a BCR-ABL level $\leq 10 \%$ did not have significantly better OS than those who had a BCR-ABL level $>10 \%$ at three months $(100.0 \%$ vs. $95.2 \%$, respectively; $p=0.545)$. There was no difference in the OS of patients who had $<1$ log BCR-ABL reduction and those who had $\geq 1 \log$ BCR-ABL reduction at 12 months $(91.3 \%$ vs. $100.0 \%$, respectively; $\mathrm{p}=0.142$ ). 
Table II. Outcomes following treatment with imatinib.

\begin{tabular}{|c|c|c|c|c|}
\hline Treatment outcome & OS (\%) & $\mathrm{p}$-value & EFS (\%) & p-value \\
\hline CCyR & & $<0.001$ & & 0.002 \\
\hline Yes & 98.2 & & 96.4 & \\
\hline No & 75.0 & & 73.0 & \\
\hline Time to achieve CCyR & & 0.637 & & 0.319 \\
\hline$\leq 12 \mathrm{mth}$ & 100.0 & & 100.0 & \\
\hline$>12 \mathrm{mth}$ & 95.8 & & 91.7 & \\
\hline MMR & & 0.012 & & 0.003 \\
\hline Yes & 97.8 & & 97.8 & \\
\hline No & 87.1 & & 92.9 & \\
\hline Time to achieve MMR & & 0.190 & & 0.366 \\
\hline$\leq 18 \mathrm{mth}$ & 100.0 & & 100.0 & \\
\hline$>18 \mathrm{mth}$ & 97.8 & & 96.2 & \\
\hline \multicolumn{5}{|l|}{ BCR-ABL } \\
\hline At 3rd mth & & 0.545 & & 0.08 \\
\hline$\leq 10 \%$ & 100.0 & & 100.0 & \\
\hline$>10 \%$ & 95.2 & & 66.7 & \\
\hline At 6 th $\mathrm{mth}$ & & 0.041 & & 0.013 \\
\hline$\leq 10 \%$ & 100.0 & & 100.0 & \\
\hline$>10 \%$ & 88.9 & & 82.4 & \\
\hline At 12 th $\mathrm{mth}$ & & 0.142 & & 0.006 \\
\hline$\geq 1$ log reduction & 100.0 & & 100.0 & \\
\hline$<1$ log reduction & 91.3 & & 81.8 & \\
\hline
\end{tabular}

CCyR: complete cytogenetic response; EFS: event-free survival at ten years; MMR: major molecular response; OS: overall survival at ten years

The ten-year event-free survival (EFS) among the 70 patients was $92.9 \%$. The EFS was better among the patients who achieved CCYR and MMR than those who did not (CCyR: $96.4 \%$ vs. $73.0 \%$, respectively; $p=0.002$ and MMR: $97.8 \%$ vs. $92.9 \%$, respectively; $\mathrm{p}=0.003)$. The EFS was also better among the patients who had a BCR-ABL level $\leq 10 \%$ at six months than those who had a BCRABL level $>10 \%$ at six months $(100.0 \%$ vs. $82.4 \%$, respectively; $p=0.013)$. There was no significant difference in the EFS of the patients who achieved MMR at or before 18 months and the EFS of those who did not $(100.0 \%$ vs. $96.2 \%$, respectively; $p=0.366)$. Similarly, there was no significant difference in the EFS of the patients who achieved CCYR at or before 12 months and those who did not $(100.0 \%$ vs. $91.7 \%$, respectively; $p=0.319)$. On the other hand, we found that $\mathrm{a}<1$ log reduction of $\mathrm{BCR}-\mathrm{ABL}$ at 12 months predicts poorer outcome; the EFS was $81.8 \%$ and $100.0 \%$ for patients who had $<1$ log reduction of BCR-ABL and those who had $\geq 1 \log$ reduction of BCR-ABL at 12 months, respectively $(p=0.006)$.

\section{DISCUSSION}

The survival of patients with CML has improved significantly since the introduction of imatinib more than a decade ago. Early-phase CML patients receiving imatinib can have an almost-normal lifespan. The IRIS trial showed that the eight-year survival rate for CML patients on imatinib was $85 \%$; the survival rate was even better (i.e. $93 \%$ ) if only CML-related deaths were considered. ${ }^{(2,3)}$ In the present study, the patients on imatinib showed a similarly excellent survival rate, with a median survival duration of

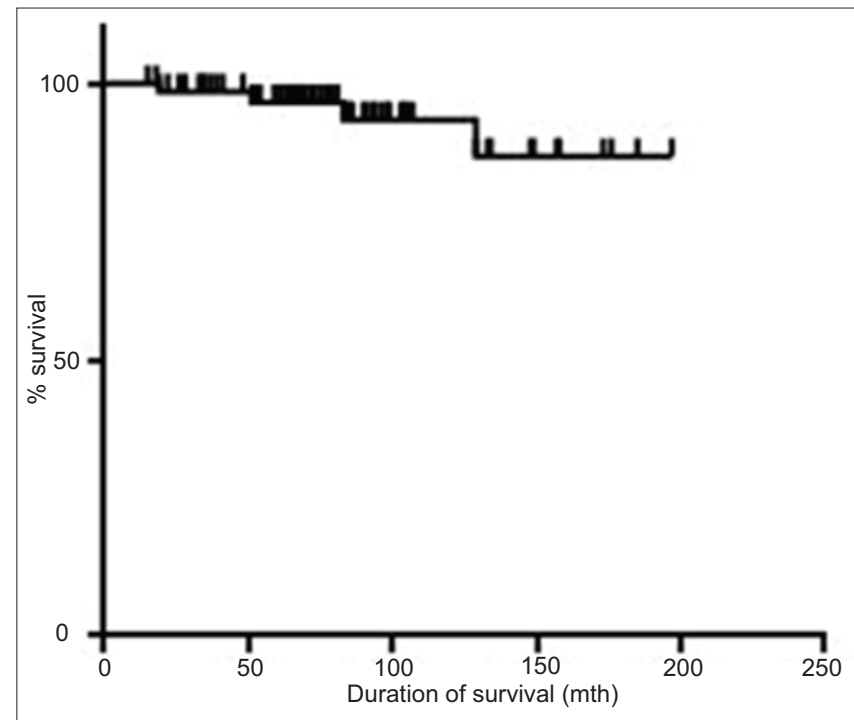

Fig. 1 Graph shows the overall duration of survival of the patients $(n=70)$.

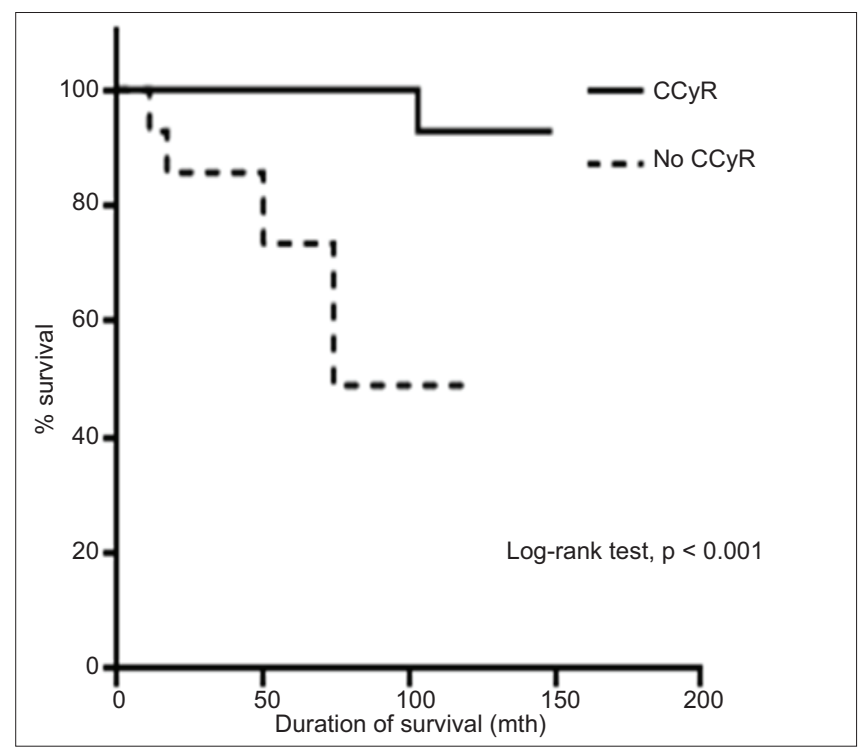

Fig. 2 Graph shows the duration of survival of the patients according to whether they achieved complete cytogenetic response (CCyR).

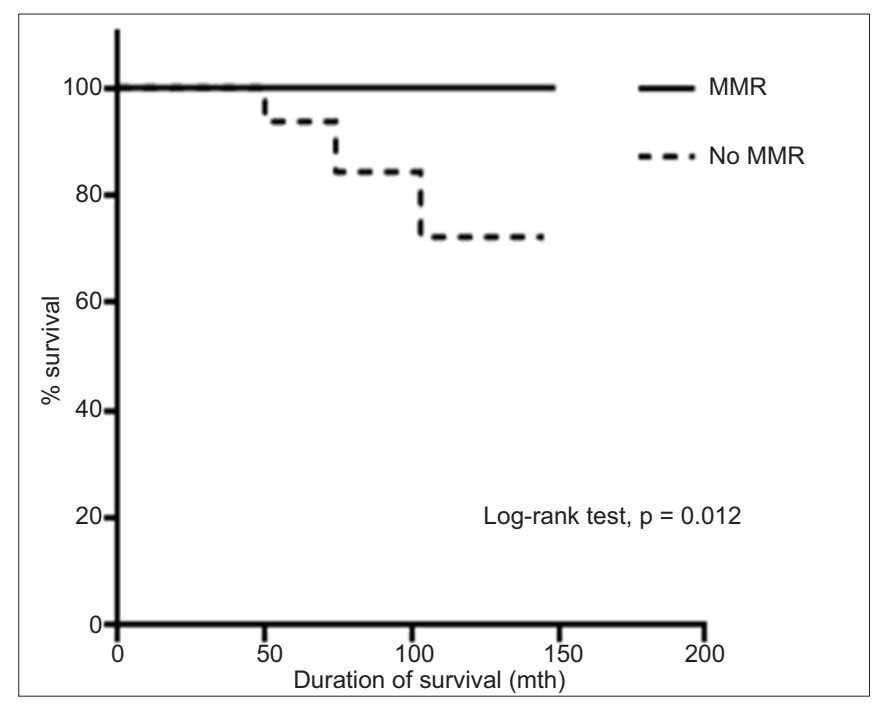

Fig. 3 Graph shows the duration of survival of the patients according to whether they achieved major molecular response (MMR). 


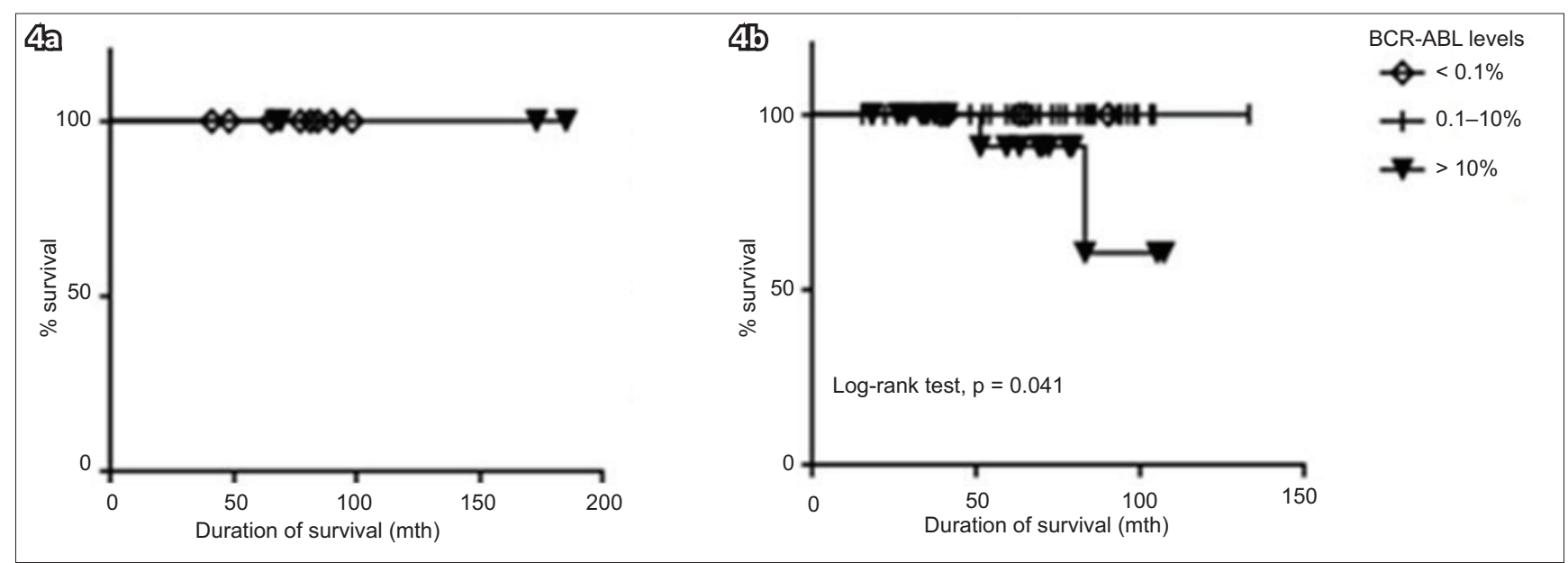

Fig. 4 Graphs show the duration of survival of the patients, according to their BCR-ABL levels at (a) three months and (b) six months.

74 (range 15-197) months and OS of $94.3 \%$. In the IRIS trial, long-term follow-up of the patients also revealed that nearly all the patients who remained on imatinib achieved CCyR, and progression of the disease after the first three years of treatment was extremely rare. ${ }^{(2)}$ In comparison, the percentage of CCyR and MMR among the patients in the present study was $80.0 \%$ and $65.7 \%$, respectively, which are comparable to other studies. ${ }^{(15,16)}$ Hence, the results of our study show that a good response can also be achieved in a real-life clinical setting.

Even though imatinib has been proven to have excellent efficacy, there are still a considerable number of patients who respond poorly to it. Therefore, many studies have been conducted to further improve the outcome of CML patients. Hehlmann et al compared imatinib 800 mg/day, imatinib $400 \mathrm{mg} /$ day and imatinib $400 \mathrm{mg}$ /day plus interferon to determine if more intensive treatment would result in a better outcome. ${ }^{(17)}$ The results of their study showed that patients on high-dose imatinib (i.e. $800 \mathrm{mg} /$ day) had a significantly higher incidence of MMR at 12 months. However, the better responses did not translate to better OS and PFS at three years. ${ }^{(17)}$ This observation was further confirmed in a systematic review and meta-analysis by Gafter-Gvili et al. ${ }^{(18)}$ In that review of four randomised trials, the authors showed that CCYR and MMR at 12 months were improved by high-dose imatinib in patients with chronic-phase $\mathrm{CML}$; however, there was no difference in allcause mortality or disease progression at the end of follow-up. ${ }^{(18)}$ The results of the present study revealed that the patients who achieved CCYR and MMR had better OS and EFS as compared to those who failed to achieve these responses (Table I). However, the OS and EFS did not differ between the patients who attained CCyR at or before 12 months and those who attained CCyR after 12 months, or between the patients who attained MMR at or before 18 months and those who attained MMR after 18 months. This finding is in contrast to Hughes et al's study, ${ }^{(2)}$ which showed that patients who achieved MMR at 18 months had better EFS. This difference could be due to the small sample size $(n=70)$ of the present study.

In a retrospective analysis of 1,440 newly diagnosed chronicphase CML patients, OS rates at five years were better among the patients who had a BCR-ABL level between $1 \%$ and $10 \%$ at three months (OS 94\%) and $\leq 1 \%$ (OS 97\%) at three months, as compared to the patients who had a BCR-ABL level $>10 \%$ at three months (OS 87\%). ${ }^{(19)}$ In addition, a BCR-ABL level > 1\% at six months was associated with inferior survival rates at five years when compared to a BCR-ABL level $<1 \%$ at six months (89\% vs. 97\%). ${ }^{(19)}$ Jain et al published similar evidence from their single-centre experience with 483 newly diagnosed chronicphase CML patients who received either $400 \mathrm{mg}$ or $800 \mathrm{mg}$ of imatinib, nilotinib or dasatinib as first-line treatment. ${ }^{(20)}$ The three-year failure-free survival (FFS) was better if the three-month BCR-ABL level was $\leq 1 \%$ and not between $1 \%$ and $10 \%$ or $>10 \%$ (FFS $85 \%, 73 \%$ and $61 \%$, respectively; $p=0.016$ ). The three-year FFS for the patients with a six-month BCR-ABL level $\leq 1 \%$ was $89 \%$, while it was $56 \%$ for patients with a BCR-ABL level between $1 \%$ and $10 \%$, and $49 \%$ for those with a BCR-ABL level $>10 \%(p<0.001){ }^{(20)}$

The ENESTnd (Evaluating Nilotinib Efficacy and Safety in clinical Trials-newly diagnosed patients) study, which compared the use of nilotinib and imatinib in newly diagnosed chronicphase $C M L$ patients, also reported correlations between $B C R-A B L$ levels at three months, and PFS and OS by four years. ${ }^{(9)}$ Similar results were seen in the DASISION (DASatinib versus Imatinib Study In treatment-Naive CML patients) trial, which compared the use of dasatinib and imatinib in newly diagnosed chronic-phase CML patients. ${ }^{(8)}$ At the end of three years, better PFS and OS were observed in the patients with a three-month BCR-ABL level $\leq 10 \%$ than in the patients with a three-month BCR-ABL level $>10 \%$ (PFS: $93 \%$ vs. $68 \%$, $p=0.0003$; OS: $96 \%$ vs. $86 \%, p=0.03){ }^{(8)}$ Marin et al identified that patients with a BCR-ABL level $<9.84 \%$ at three months and $<1.67 \%$ at six months had significantly higher eight-year OS than patients with higher transcript levels $(93.3 \%$ vs. $56.9 \%$ and $93.7 \%$ vs. $74.7 \%$, respectively). ${ }^{(21)}$ In contrast, a BCR-ABL level $>10 \%$ at six months predicted poorer survival in the patients in the present study; at six months, the OS for patients with a BCR-ABL level $\leq 10 \%$ and patients with a BCR-ABL level $>10 \%$ were $100.0 \%$ and $88.9 \%$, respectively $(p=0.041)$. This finding is consistent with the results published by Klamová et al, which showed that patients with a BCR-ABL level $<10 \%$ at six 
months had better survival. ${ }^{(16)}$ Furthermore, Hughes et al also proved that BCR-ABL levels of $>10 \%$ at six months and $>1 \%$ at 12 months predict inferior EFS and a higher rate of disease progression. (2) This observation is especially important for less developed countries, where the need to use higher dosages of imatinib or the need to change treatment from imatinib to newergeneration TKIs (in order to achieve faster and deeper responses) would result in a significant financial burden.

The present study also revealed that at 12 months, $\geq 1 \log$ reduction of $\mathrm{BCR}-\mathrm{ABL}$ predicts better $\mathrm{EFS}$ when compared to $<1$ log reduction of BCR-ABL (100.0\% vs. $81.8 \%$, respectively; $p=0.006)$, even though no significant difference was observed between the OS of these two groups (100\% vs. $91.3 \%, p=0.142)$.

Second-generation TKIs are more potent, and induce faster and higher rates of CCYR and molecular responses. They have also recently been approved as first-line therapy. While there is a trend of better PFS with these newer TKIs, there is no evidence to show improved OS. ${ }^{(22)}$ Imatinib is still advantageous since it is effective in a large proportion of patients. In addition, its safety profile is well established. More importantly, the imminent availability of generic imatinib could potentially reduce the financial burden of long-term therapy for CML. This is crucial, especially in developing countries, as experts have argued that the prices of cancer treatment, with CML treatment as the example, are not sustainable. ${ }^{(23)}$

In conclusion, the results of the present study show that imatinib is still useful as first-line therapy. However, vigilant monitoring of patients whose BCR-ABL level is $>10 \%$ at six months of treatment should be implemented so that prompt action can be taken to provide the best outcome for these patients.

\section{REFERENCES}

1. Druker BJ, Guilhot F, O'Brien SG, et al; IRIS Investigators. Five-year follow-up of patients receiving imatinib for chronic myeloid leukemia. N Engl J Med 2006; 355:2408-17.

2. Hughes TP, Hochhaus A, Branford S, et al; IRIS Investigators. Longterm prognostic significance of early molecular response to imatinib in newly diagnosed chronic myeloid leukemia: an analysis from the International Randomized Study of Interferon and STI571 (IRIS). Blood 2010; 116:3758-65.

3. Deininger $M, \mathrm{O}^{\prime}$ Brien $\mathrm{SG}$, Guilhot $\mathrm{F}$, et al. International randomized study of interferon vs STI571 (IRIS) 8-year follow up: sustained survival and low risk for progression or events in patients with newly diagnosed chronic myeloid leukemia in chronic phase $(\mathrm{CML}-\mathrm{CP})$ treated with imatinib. 51st ASH Annual Meeting and Exposition. Oral and Poster Abstracts. Poster Session: Chronic Myeloid Leukemia - Therapy Poster I.

4. Wang L, Pearson K, Ferguson JE, Clark RE. The early molecular response to imatinib predicts cytogenetic and clinical outcome in chronic myeloid leukaemia. Br J Haematol 2003; 120:990-9.

5. Cortes J, Talpaz M, O'Brien S, et al. Molecular responses in patients with chronic myelogenous leukemia in chronic phase treated with imatinib mesylate. Clin Cancer Res 2005; 11:3425-32.

6. Latagliata R, Isidori A, Breccia M, et al. Complete clearance of Ph+ metaphases after 3 months is a very early indicator of good response to imatinib as front-line treatment in chronic myelogenous leukemia. Acta Haematol 2013; 129:126-34.

7. Hanfstein B, Shlyakhto V, Lauseker M, et al. Velocity of early BCR-ABL transcript elimination as an optimized predictor of outcome in chronic myeloid leukemia (CML) patients in chronic phase on treatment with imatinib. Leukemia 2014; 28:1988-92.

8. Kantarjian H, Shah NP, Hochhaus A, et al. Dasatinib versus imatinib in newly diagnosed chronic-phase chronic myeloid leukemia. N Engl J Med 2010; 362:2260-70.

9. Saglio G, Kim DW, Issaragrisil S, et al; ENESTnd Investigators. Nilotinib versus imatinib for newly diagnosed chronic myeloid leukemia. $\mathrm{N}$ Engl J Med 2010; 362:2251-9.

10. Hochhaus A, Baccarani M, Deininger M, et al. Dasatinib induces durable cytogenetic responses in patients with chronic myelogenous leukemia in chronic phase with resistance or intolerance to imatinib. Leukemia 2008; 22:1200-6.

11. Kantarjian H, Pasquini R, Lévy V, et al. Dasatinib or high-dose imatinib for chronic-phase chronic myeloid leukemia resistant to imatinib at a dose of 400 to 600 milligrams daily: two-year follow-up of a randomized phase 2 study (START-R). Cancer 2009; 115:4136-47.

12. Jabbour E, Kantarjian H, Cortes J. Chronic myeloid leukemia and secondgeneration tyrosine kinase inhibitors: when, how, and which one? Semin Hematol 2010; 47:344-53.

13. O'Brien S, Abboud CN, Akhtari M, et al; National Comprehensive Cancer Network. Chronic myelogenous leukemia. J Natl Compr Canc Netw 2012; 10:64-110.

14. Baccarani M, Deininger MW, Rosti G, et al. European LeukemiaNet recommendations for the management of chronic myeloid leukemia: 2013. Blood 2013; 122:872-84.

15. Bendit I, Sanabani SS, Conchon M, et al. Evaluation of long-term outcomes, cytogenetic and molecular responses with imatinib mesylate in early and late chronic-phase chronic myeloid leukemia: a report from a single institute. Acta Haematol 2012; 128:223-32.

16. Klamová H, Poláková KM, Mužík J, et al. Evaluation of 5-year imatinib treatment of 458 patients with CP-CML in routine clinical practice and prognostic impact of different BCR-ABL cutoff levels. Cancer Med 2013; 2:216-25.

17. Hehlmann R, Lauseker M, Jung-Munkwitz S, et al. Tolerability-adapted imatinib $800 \mathrm{mg} / \mathrm{d}$ versus $400 \mathrm{mg} / \mathrm{d}$ versus $400 \mathrm{mg} / \mathrm{d}$ plus interferon- $\alpha$ in newly diagnosed chronic myeloid leukemia. J Clin Oncol 2011; 29:1634-42.

18. Gafter-Gvili A, Leader A, Gurion R, et al. High-dose imatinib for newly diagnosed chronic phase chronic myeloid leukemia patients--systematic review and meta-analysis. Am J Hematol 2011; 86:657-62.

19. Hanfstein B, Müller MC, Hehlmann R, et al; SAKK; German CML Study Group. Early molecular and cytogenetic response is predictive for longterm progression-free and overall survival in chronic myeloid leukemia (CML). Leukemia 2012; 26:2096-102.

20. Jain $P$, Kantarjian $H$, Nazha A, et al. Early responses predict better outcomes in patients with newly diagnosed chronic myeloid leukemia: results with four tyrosine kinase inhibitor modalities. Blood 2013; 121:4867-74.

21. Marin D, Ibrahim AR, Lucas C, et al. Assessment of BCR-ABL1 transcript levels at 3 months is the only requirement for predicting outcome for patients with chronic myeloid leukemia treated with tyrosine kinase inhibitors. J Clin Oncol 2012; 30:232-8.

22. Hughes TP, Saglio G, Kantarjian HM, et al. Early molecular response predicts outcomes in patients with chronic myeloid leukemia in chronic phase treated with frontline nilotinib or imatinib. Blood 2014; 123:1353-60.

23. Experts in Chronic Myeloid Leukemia. The price of drugs for chronic myeloid leukemia (CML) is a reflection of the unsustainable prices of cancer drugs: from the perspective of a large group of CML experts. Blood 2013; 121:4439-42. 